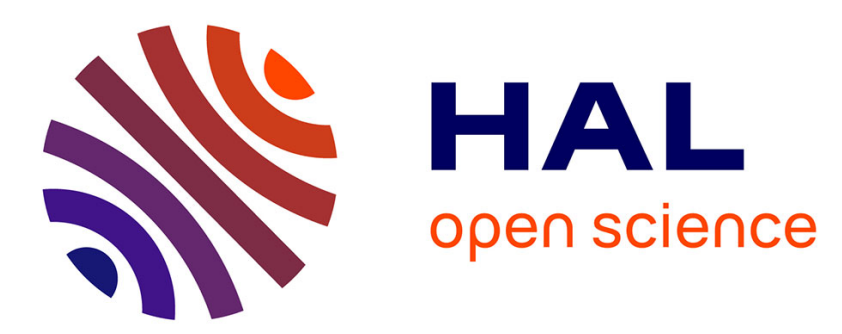

\title{
State-Dependent Sampling for Linear Time Invariant Systems: A Discrete Time Analysis
}

Sonia Maalej, Christophe Fiter, Laurentiu Hetel, Jean-Pierre Richard

\section{To cite this version:}

Sonia Maalej, Christophe Fiter, Laurentiu Hetel, Jean-Pierre Richard. State-Dependent Sampling for Linear Time Invariant Systems: A Discrete Time Analysis. 20th Mediterranean Conference on Control \& Automation (MED), Jul 2012, Barcelone, Spain. pp.1129 - 1134. hal-00733212

\section{HAL Id: hal-00733212 \\ https://hal.science/hal-00733212}

Submitted on 18 Sep 2012

HAL is a multi-disciplinary open access archive for the deposit and dissemination of scientific research documents, whether they are published or not. The documents may come from teaching and research institutions in France or abroad, or from public or private research centers.
L'archive ouverte pluridisciplinaire HAL, est destinée au dépôt et à la diffusion de documents scientifiques de niveau recherche, publiés ou non, émanant des établissements d'enseignement et de recherche français ou étrangers, des laboratoires publics ou privés. 


\title{
State-Dependent Sampling for Linear Time Invariant Systems: A Discrete Time Analysis
}

\author{
Sonia MAALEJ, Christophe FITER, Laurentiu HETEL, Jean-Pierre RICHARD
}

\begin{abstract}
This work concerns the adaptation of sampling times for Linear Time Invariant (LTI) systems controlled by state feedback. Complementary to various works that guarantee stabilization independently of changes in the sampling rate, here we provide conditions to design stabilizing sequences of sampling instants. In order to reduce the number of these sampling instants, a dynamic scheduling algorithm optimizes, over a given sampling horizon, a sampling sequence depending on the system state value. Our proofs are inspired by switched system techniques combining Lyapunov functions and LMI optimization. To show the applicability of the technique, the theoretical study is illustrated by an implementation in Matlab/TRUE TIME.

Index Terms - Embedded Systems, Switching Systems, Sampling-Data Systems, Real-Time Control, Scheduling, Stability, Hybrid Dynamic Systems, Linear Matrix Inequalities (LMI).
\end{abstract}

\section{INTRODUCTION}

Real-time control concerns both automation and information sciences. It implies the interaction of the control task with the real-time scheduling for sampling. Generally, the scheduling mechanism manages the execution of a tasks set and the resources linked to a computer system (CPU, communication network, router, ...). These resources are often limited and their availability is variable, which may lead to performance degradation or loss of stability [1], [2]. As a result, much attention has been directed to embedded and/or networked-control systems [3] [4].

Several studies have addressed issues related to delays (due to communication or to the resources access) and to the sampling effect which is not necessarily periodic. The problem of robustness regarding variable sampling [5], [6], [7], [8], variable delay [9], [10], [11], or the combination of the two problems [12], [13], [14], attracted a considerable interest. All these works present robust stability conditions provided that the sampling step or the delay remains below some upper bounds.

The research leading to these results has received funding from the European Community's 7th Framework Programme (FP7/2007-2013) grant agreement No 257462 HYCON2 Network of Excellence.

S. Maalej, C. Fiter, L. Hetel and J-P. Richard are with the Ecole Centrale de Lille, LAGIS (Laboratoire d'Automatique, Génie Informatique et Signal, CNRS UMR 8219), 59651 Villeneuve d'Ascq, France sonia.maalej@ec-lille.fr

S. Maalej and J-P Richard are with the Non-A, INRIA Lille-Nord Europe, France
The proofs are based on Lyapunov functions leading to Linear Matrix Inequalities (LMI). The provided upper-bounds are not depending on the state value.

In [2], it is considered a periodic sequence of sampling times, corresponding to an offline static scheduling and, again, the sampling rate is defined independently of the position in the state space.

In parallel to the robustness issue, recent works also consider the resource issue, linked to the reduction of the sampling instants. Two main approaches are distinguished: event-triggered sampling [15], [16], [17], [18], [19], and self-triggered sampling [20], [21], [22], [23]. In the first case, information is sent to the controller when special events happen (for example, crossing a border in the state space). This requires a dedicated control hardware. In the second approach, the self-triggered controller emulates the event-triggered controller, but without using dedicated hardware. This means that at each sampling instant, one calculates the lower bound approximation of the next admissible sampling interval.

More recently, [24] proposed to compute offline a mapping of a sampling function of the state space: each region of the state partition is associated to an acceptable maximum sampling step. This technique is based on a dynamic hybrid model. Once again, this study involves Lyapunov functions that ensure the continuous-time system stability under sampling with variable steps. The offline computation constitutes a main advantage.

This work follows [24] and proposes a different statedependent sampling approach. The novelty compared to [24] is to consider the optimization over a discrete set of sequences corresponding to a finite sampling horizon. It leads to an implicit mapping of the state space based on Lyapunov functions.

This paper is organized as follows. Section 2 presents the problem and proposes a switching system model over a given sampling horizon. Then Section 3 develops the dynamic scheduling algorithm for determining future sampling steps. Finally, the theoretical study is illustrated in Section 4 and Section 5 gives concluding remarks. 


\section{Problem formulation}

A. Ideal model of a continuous process controlled by state feedback

We address LTI systems such as:

$$
\dot{x}(t)=A_{c} x(t)+B_{c} u(t), t>0 ; x(0) \in \mathbb{R}^{n},
$$

with $A_{c} \in \mathbb{R}^{n \times n}$ the state matrix, $B_{c} \in \mathbb{R}^{n \times r}$ the input matrix, $x(t) \in \mathbb{R}^{n}$ the state of the continuous system, and $u(t) \in \mathbb{R}^{r}$ the system control.

Hypothesis 1: The system (1) is supposed to be controllable. Thus, there exists a state feedback gain $K \in \mathbb{R}^{r \times n}$ :

$$
u(t)=K x(t)
$$

such that the system:

$$
\dot{x}(t)=\left(A_{c}+B_{c} K\right) x(t)
$$

is asymptotically stable, i.e. the matrix $\left(A_{c}+B_{c} K\right)$ is Hurwitz.

B. Model of a process with a digital controller

The sampling control loop is described in Fig. 1.

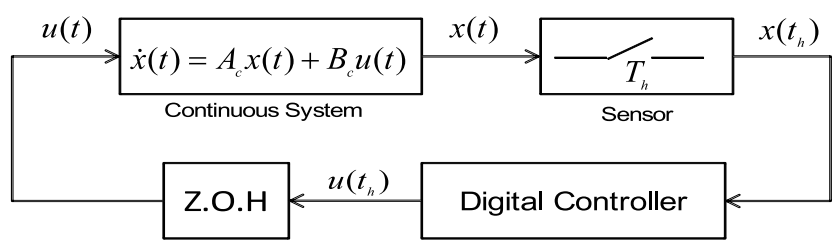

Fig. 1. Digital control diagram

We suppose that the state $x(t)$ of the system (3) is sampled at instants $t_{h}$ with $h \in \mathbb{N}$, such as $t_{h+1}>t_{h}$, $t_{0}=0$ and $t_{h} \stackrel{h \rightarrow \infty}{\longrightarrow} \infty$. The sampling intervals are denoted $T_{h}=t_{h+1}-t_{h}, h \in \mathbb{N}$. The sampled system state $x\left(t_{h}\right)$ leads to the control $u(t)=K x\left(t_{h}\right), \forall t \in\left[t_{h}, t_{h+1}\right)$. Thus, the discretized model of the system at instants $t_{h}$ is:

$x\left(t_{h+1}\right)=e^{A_{c} T_{h}} x\left(t_{h}\right)+\int_{0}^{T_{h}} e^{A_{c} s} d s B_{c} u\left(t_{h}\right), h \in \mathbb{N}$.

Defining $A_{d_{\left(T_{h}\right)}}=e^{A_{c} T_{h}}$ and $B_{d_{\left(T_{h}\right)}}=\int_{0}^{T_{h}} e^{A_{c} s} d s B_{c}$, leads to the discrete-time form:

$$
x\left(t_{h+1}\right)=\left(A_{d_{\left(T_{h}\right)}}+B_{d_{\left(T_{h}\right)}} K\right) x\left(t_{h}\right)=\tilde{A}_{\left(T_{h}\right)} x\left(t_{h}\right),
$$

where $h \in \mathbb{N}$ and $\tilde{A}_{\left(T_{h}\right)}=A_{d_{\left(T_{h}\right)}}+B_{d_{\left(T_{h}\right)}} K$.

\section{Motivation}

This work aims at characterizing sampling sequences $T_{h}$ that stabilize the system. When the sampling interval is constant, $T_{h}=T$, the system is stable if $\tilde{A}_{(T)}$ is a Schur matrix.

Definition 1: If the matrix $\tilde{A}_{(T)}$ is a Schur matrix, we call $T$ a stabilizing sampling step. Otherwise, it is called a non stabilizing sampling step.
Definition 2: A periodic sampling sequence of length $l$ is a sequence $\left\{T_{h}\right\}_{h \in \mathbb{N}}$ such that $T_{h+l}=T_{h}, \forall h \in \mathbb{N}$.

Based on [2], note that the sampled system may be asymptotic stable with a combination of stabilizing and non stabilizing sampling steps. For example, consider:

$$
A_{c}=\left(\begin{array}{ll}
1 & 3 \\
2 & 1
\end{array}\right), B_{c}=\left(\begin{array}{c}
1 \\
0.6
\end{array}\right) \text { and } K=\left(\begin{array}{ll}
-1 & -6
\end{array}\right) .
$$

Fig. 2 shows that the asymptotic stability interval of the system discretized with a constant sampling interval is: $T \in\left[T_{\min }, T_{\max }\right]=[0,0.59]$.

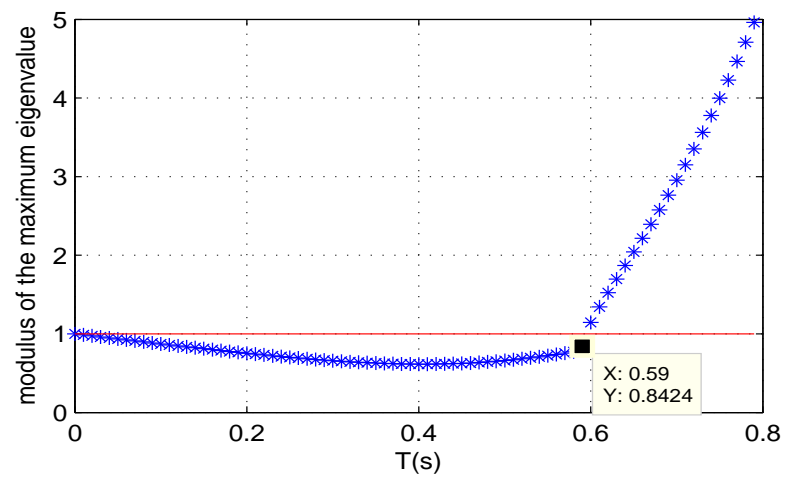

Fig. 2. Evolution of the modulus of the maximum eigenvalue $\tilde{A}_{(T)}$ in terms of $T$

Consider a periodic sampling sequence of length-two, $\left\{T_{h}\right\}_{h \in \mathbb{N}}$, with elements $T_{a}$ and $T_{b}$. The stability domain for a periodic sampling sequence of the type: $\left(T_{a}, T_{b}, T_{a}, T_{b}, \ldots\right)$ is calculated by analyzing the transition matrix $\tilde{A}_{\left(T_{a}\right)} \tilde{A}_{\left(T_{b}\right)}$ over a period. If the product matrix $\tilde{A}_{\left(T_{a}\right)} \tilde{A}_{\left(T_{b}\right)}$ is a Schur matrix, then the system is asymptotically stable. We present in Fig. 3 the stability domain for system (3) with parameters given by (6).

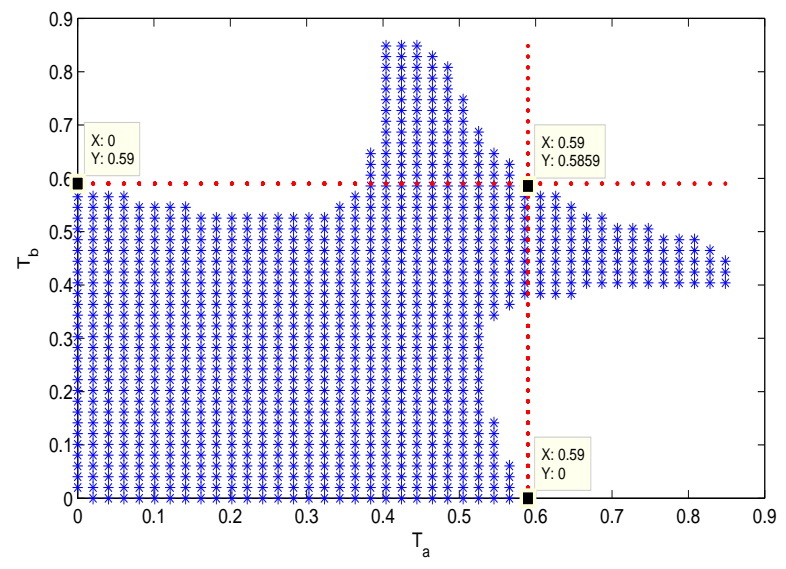

Fig. 3. Stability domain for a periodic sampling sequence: $\left(T_{a}, T_{b}, T_{a}, T_{b}, \ldots\right)$

From this figure, we can see that there are sampling sequences $\left(T_{a}, T_{b}, T_{a}, T_{b}, \ldots\right)$ such as $T_{a}=0.8081$ and $T_{b}=0.4848$, which are stabilizing the system despite the 
fact that the sampling time $T_{a}>T_{\max }$ (a simulation with this sampling sequence is presented in Fig. 4).
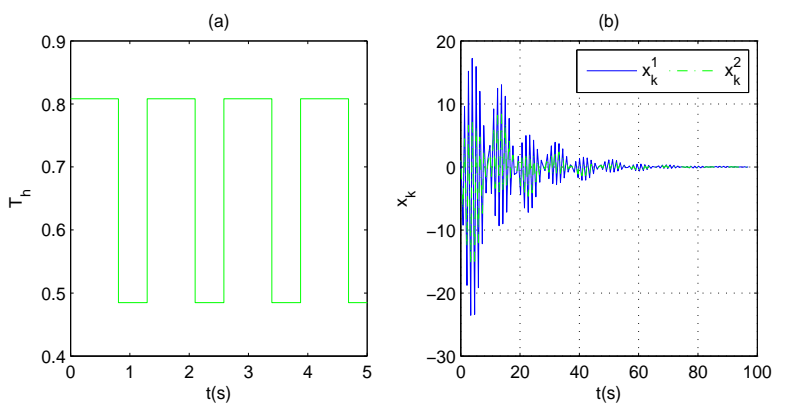

Fig. 4. Variation of the system state (5) discretized by the periodic sequence $(0.8081,0.4848,0.8081,0.4848, \ldots)$

Motivated by this observation, this article aims to characterize other sampling sequences that stabilize the system (5) by using sampling intervals higher than $T_{\max }$. Here, we shall not limit the study to periodic sampling case since the sampling sequences will depend on the system state. For this reason, we try to characterize the evolution of sampling sequences over a finite horizon (which will be denoted $\sigma$ ). The sampling horizon is formed by several sampling steps and it evolves according to the system's state.

\section{Mathematical formulation}

For $l \in \mathbb{N}^{*}, \sigma=\left\{T^{j}\right\}_{j=1}^{l}$ refers to a sampling horizon of length $l$, where $j$ represents the position of a sampling inside the horizon. Consider $\Gamma$ a subset of $\mathbb{R}^{+}$.

We define $S_{l_{\min }}^{l_{\max }}(\Gamma)$ the set of all horizons $\sigma=\left\{T^{j}\right\}_{j=1}^{l}$ of length $l \stackrel{l_{\min }}{\in}\left[l_{\min }, l_{\max }\right], T^{j} \in \Gamma$ where $l_{\min }$ and $l_{\text {max }}$ represent respectively the minimum and maximum lengths of horizons $\sigma \in S_{l_{\min }}^{l_{\max }}(\Gamma)$.

By extension, we note $S_{l_{\min }}^{l_{\max }}$ the set of all sampling horizons with values in $\mathbb{R}^{+}$and length within the interval $\left[l_{\text {min }}, l_{\text {max }}\right]$.

We denote $\sigma_{k}=\left(T_{k}^{1}, T_{k}^{2}, \ldots, T_{k}^{l_{k}}\right) \in S_{l_{\min }}^{l_{\max }}$ with $k \in \mathbb{N}$, a sampling horizon sequence. $T_{k}^{i}$ with $k \in \mathbb{N}$ and $i \in$ $\left\{1, \ldots, l_{k}\right\}$, define sampling steps where $k$ indicates the index of the horizon and $i$ the position of this sampling step in the considered horizon $\sigma_{k}$.

We consider then $\Theta=\left\{T_{h}\right\}_{h \in \mathbb{N}}$ a sampling sequence characterized by the concatenation of horizons $\left\{\sigma_{k}\right\}_{k \in \mathbb{N}} \in S_{l_{\min }}^{l_{\max }}$. Hence:

$$
(\underbrace{T_{0}^{1}, T_{0}^{2}, \ldots, T_{0}^{l_{0}}}_{\sigma_{0}}, \underbrace{T_{1}^{1}, T_{1}^{2}, \ldots, T_{1}^{l_{1}}}_{\sigma_{1}}, \ldots, \underbrace{T_{k}^{1}, T_{k}^{2}, \ldots, T_{k}^{l_{k}}}_{\sigma_{k}}, \ldots) .
$$

Finally, we note $\left.\tau_{k}\right|_{k \in \mathbb{N}}$ the starting time of an horizon $\sigma_{k}$ such as $\tau_{k+1}=\tau_{k}+\sum_{i=1}^{l_{k}} T_{k}^{i}$ and $\tau_{0}=t_{0}=0$ (see Fig. 5).

The representation of system (5) over a sampling horizon for a sequence $\sigma_{k}$ is given as follows:

$$
x_{k+1}=\Phi_{\sigma_{k}} x_{k}, k \in \mathbb{N},
$$

where $x_{k}=x\left(\tau_{k}\right)$ the system state at the starting time $\tau_{k}$ of the horizon $\sigma_{k}$ (see Fig. 6), and $\Phi_{\sigma_{k}}=\tilde{A}_{\left(T_{k}^{l} l_{k}\right)} \tilde{A}_{\left(T_{k}^{l_{k}-1}\right)} \ldots \tilde{A}_{\left(T_{k}^{1}\right)}$ the transition matrix corresponding to the sequence of the sampling horizon $\sigma_{k}$, from the instant $\tau_{k}$ to the instant $\tau_{k+1}$.

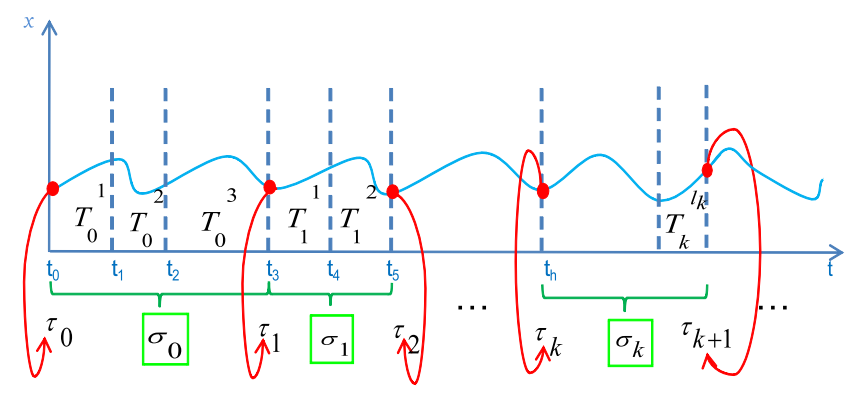

Fig. 5. System state discretization using variable sequences

Our aim is to calculate, at instant $\tau_{k}$ the next sampling horizon $\sigma_{k}$ which will be applied to the sampling mecanism so to ensure stability. From equation (7), we remark that this objective amounts to a problem of controlling a linear switching system [25], [26]. In fact, switched systems constitute a class of hybrid systems for which a discrete-event law shows an active subsystem from a family of continuous dynamic subsystems [27], [28].

Our mathematical problem can be exposed as follows:

Problem : For sampling steps $T_{h} \in \Gamma$, determine the sampling horizon evolution according to the system state (7) at instant $\tau_{k}$ of the horizon start, i.e. define $\sigma_{k}$ as a function $f\left(x\left(\tau_{k}\right)\right.$ ) (such that $\sigma_{k}=f\left(x\left(\tau_{k}\right)\right)$ ) in order to guarantee the asymptotic stability.

\section{Results}

This section presents the main theorem which allows us to provide sampling functions that guarantee the stability of the system (1) with a sampled control $u(t)=$ $K x\left(t_{h}\right)$. Next, we describe an algorithm generating the corresponding sampling law.

\section{A. Stability Theorem}

To ensure the asymptotic stability of the discretized system (7), we control the switching law $\sigma_{k}$ defining the sampling step sequences using Theorem 1. A diagram is shown in Fig. 6.

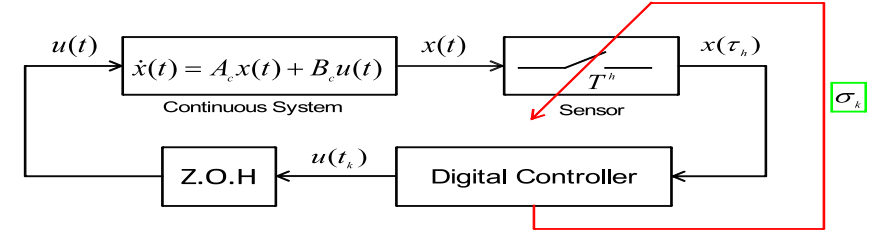

Fig. 6. Block diagram of the state-dependent sampled control 
Theorem 1:

Consider system (7) and let:

$\bar{S}_{l_{\text {min }}}^{l_{\text {max }}}=\left\{\sigma \in S_{l_{\text {min }}}^{l_{\text {max }}}, \exists P_{\sigma}=P_{\sigma}^{T}>0 ; \Phi_{\sigma}^{T} P_{\sigma} \Phi_{\sigma}-P_{\sigma}<0\right\}$

be the subset of stable periodic sequences in the set $S_{l_{\min }}^{l_{\max }}$, such that, for each $\sigma \in \bar{S}_{l_{\min }}^{l_{\max }}, P_{\sigma}=P_{\sigma}^{T}>0$ satisfies the following LMI:

$$
\Phi_{\sigma}^{T} P_{\sigma} \Phi_{\sigma}-P_{\sigma}<0 .
$$

Consider the function $f: \mathbb{R}^{n} \longrightarrow \bar{S}_{l_{\min }}^{l_{\max }}$ defined by:

$$
f(x)=\operatorname{argmin}_{i \in \bar{S}_{l_{\min }^{\max }}^{\max }}\left(x^{T} \Phi_{i}^{T} P_{i} \Phi_{i} x\right) ; x \in \mathbb{R}^{n} .
$$

Then the system (7) with $\sigma_{k}=f\left(x_{k}\right)$, i.e:

$$
x_{k+1}=\Phi_{\sigma_{k}} x_{k},
$$

is asymptotically stable.

Proof 1: By applying Lemma 2 from [26] to system (10), a sufficient condition for the system stability is ensured by the existence of a matrix series $\widetilde{P}_{k} \in \mathbb{R}^{n \times n}$ and scalars $\alpha, \beta, \gamma>0$ such that $\forall k \geq 0$ :

$$
\alpha I \leq \widetilde{P}_{k} \leq \beta I \text { and } \Phi_{\sigma_{k}}^{T} \widetilde{P}_{k+1} \Phi_{\sigma_{k}}-\widetilde{P}_{k} \leq-\alpha I .
$$

This implies that there exists a strictly decreasing function $V\left(x_{k}, k\right)=x_{k}^{T} \widetilde{P}_{k} x_{k}$. Note that this function depends both on the state $x_{k}$ and on the time $t_{k}$.

In this paper, we consider particularly the poly-quadratic Lyapunov function $V: \mathbb{R}^{n} \times \mathbb{N} \longrightarrow \mathbb{R}^{+}$defined by:

$$
V\left(x_{k}, k\right)= \begin{cases}x_{0}^{T} P_{\sigma_{0}} x_{0} & k=0 \\ x_{k}^{T} P_{\sigma_{k-1}} x_{k} & k>0\end{cases}
$$

with $P_{\sigma}=P_{\sigma}^{T} \succ 0$ as $\sigma \in \bar{S}_{l_{\min }}^{l_{\max }}$.

The Lyapunov increment is:

$$
\Delta V_{k}=V\left(x_{k+1}, k+1\right)-V\left(x_{k}, k\right)
$$

- For $k=0$ :

$$
\left.\Delta V_{k}\right|_{k=0}=x_{1}^{T} P_{\sigma_{0}} x_{1}-x_{0}^{T} P_{\sigma_{0}} x_{0} .
$$

From the equation (7) we have:

$$
\left.\Delta V_{k}\right|_{k=0}=x_{0}^{T}\left(\Phi_{\sigma_{0}}^{T} P_{\sigma_{0}} \Phi_{\sigma_{0}}-P_{\sigma_{0}}\right) x_{0} .
$$

Thus, applying the inequality (8), we have, $\forall x_{0} \neq 0$, $\left.\Delta V_{k}\right|_{k=0}<0$.

- For $k>0$ :

The Lyapunov increment is:

$$
\Delta V_{k}=x_{k+1}^{T} P_{\sigma_{k}} x_{k+1}-x_{k}^{T} P_{\sigma_{k-1}} x_{k} .
$$

Thus, from (7):

$$
\Delta V_{k}=x_{k}^{T}\left(\Phi_{\sigma_{k}}^{T} P_{\sigma_{k}} \Phi_{\sigma_{k}}-P_{\sigma_{k-1}}\right) x_{k} .
$$

Moreover, the equation (9) implies that $\forall k \in \mathbb{N}$ :

$$
x_{k}^{T} \Phi_{\sigma_{k}}^{T} P_{\sigma_{k}} \Phi_{\sigma_{k}} x_{k} \leq x_{k}^{T} \Phi_{\sigma_{k-1}}^{T} P_{\sigma_{k-1}} \Phi_{\sigma_{k-1}} x_{k} .
$$

Therefore, using equations (16) and (17), we conclude that $\forall x_{k} \neq 0$ :

$$
\Delta V_{k} \leq x_{k}^{T}\left(\Phi_{\sigma_{k-1}}^{T} P_{\sigma_{k-1}} \Phi_{\sigma_{k-1}}-P_{\sigma_{k-1}}\right) x_{k} .
$$

Thus, from (8), $\Delta V_{k} \prec 0, \forall x_{k} \neq 0$.

Therefore, the system (10) is asymptotically stable.

Remark 1: One of the advantages of the discrete time approach is the fact that it determines implicitly a mapping of the state space based on Lyapunov functions.

Remark 2: Given the fact that (1) is an LTI system and that $\Gamma$ is a bounded subset, $x(t)$ can be written as:

$$
x(t)=\Lambda\left(t-\tau_{k}\right) x_{k}, \forall t \in\left[\tau_{k}, \tau_{k+1}\right)
$$

where $\Lambda$ is a linear bounded operator representing the system transition matrix from the instant $\tau_{k}$ to the instant $t$.

In this case, using similar arguments as in Proposition 2 from [29], the convergence (to zero) of the sequences $\left\|x\left(\tau_{k}\right)\right\|_{k \rightarrow \infty}$ implies the convergence (to zero) of $\|x(t)\|$ for $t \rightarrow \infty$.

\section{B. Determination of sampling law}

The following algorithm provides a switching function as in Theorem 1 which will be used as a sampling law for the sampled date system (5):

\section{Algorithm 1: \\ OFFLINE}

1) Define a finite set $\Gamma=\left\{\Gamma_{1}, \Gamma_{2}, \ldots, \Gamma_{m}\right\}$ of allowed sampling steps $\Gamma_{i} \in \mathbb{R}^{+}, \forall i \in\{1, \ldots, m\}$;

2 ) For given lengths $l_{\min }$ and $l_{\max }$ defined by the user, search $\bar{S}_{l_{\min }}^{l_{\max }}(\Gamma) \subset \bar{S}_{l_{\min }}^{l_{\max }}$, the subsets of stable sampling horizons $S_{l_{\min }}^{l_{\max }}$;

3) Compute, for each horizon $\sigma \in \bar{S}_{l_{\min }}^{l_{\max }}(\Gamma)$, a Lyapunov matrix $P_{\sigma}$ using the LMI (8);

\section{ONLINE}

4) Compute the switching law (9) in Theorem 1.

Remark 3: The cost introduced by the calculation of the switching law (9) (Step 4 of Algorithm 1) is given by:

$$
\underbrace{n(n+1) \mid \bar{S}_{l_{\min }^{\max }}^{l_{\text {max }} \mid}}_{\text {multiplications number }}+\underbrace{(n-1)(n+1)\left|\bar{S}_{l_{\min }}^{l_{\max }}\right|}_{\text {additions number }} .
$$

Thus, the complexity of the online algorithm is: $O\left(n^{2}\left|\bar{S}_{l_{\min }}^{l_{\max }}\right|\right)$ such that $\left|\bar{S}_{l_{\min }}^{l_{\max }}\right|$ denotes the cardinal of the set $\bar{S}_{l_{\min }}^{l_{\max }}$.

\section{EXAMPLE}

To better understand the application of the Algorithm 1 , we consider the system (10) described by the matrices (6) and the set of sampling step values $\Gamma=\{0.1,0.2,0.3,0.4,0.5,0.6,0.7,0.8\}$. Despite the fact that the range of stability is $\left[T_{\min }, T_{\max }\right]=[0,0.59]$, note that we choose here the set $\Gamma$ which admits sampling steps larger than $T_{\max }$.

Fig. 7 presents the sampling sequence $\Theta$ formed by the sampling horizons $\sigma_{k}$ with length $l_{k} \in\left\{l_{\min }, \ldots, l_{\max }\right\}$ with $l_{\min }=1, l_{\max }=3$ and $k \in \mathbb{N}$. 


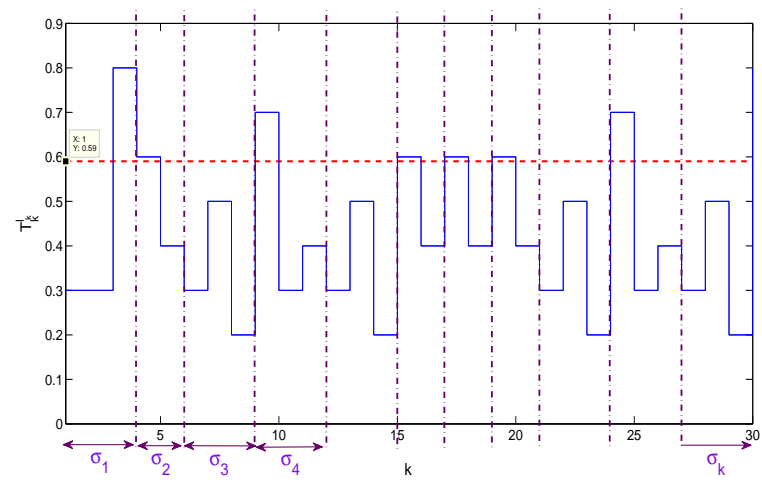

Fig. 7. Variation of sampling steps $\left.T_{k}^{i}\right|_{i \in\left\{1, \ldots, l_{k}\right\}}$

We observe that the sampling sequence is variable and that the sampling horizons have different lengths $l_{k} \in\left\{l_{\min }, \ldots, l_{\max }\right\}$. Moreover, we can see that non stabilizing sampling steps, i.e. higher than $T_{\max }$, are used to ensure the asymptotic stability of the system (see the convergence on Fig. 8).

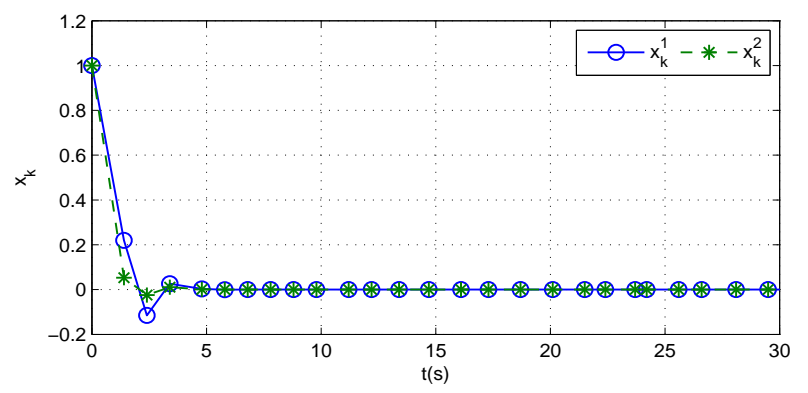

Fig. 8. Evolution of the system state using variable sampling steps and horizons $\sigma_{k}$ from Fig. 7

The stability of this system can be checked by using Theorem 1. Fig. 9 shows the evolution of the Lyapunov function $V\left(x_{k}, k\right)=x_{k}^{T} P_{\sigma_{k-1}} x_{k}$ which is positive and decreasing over the time.

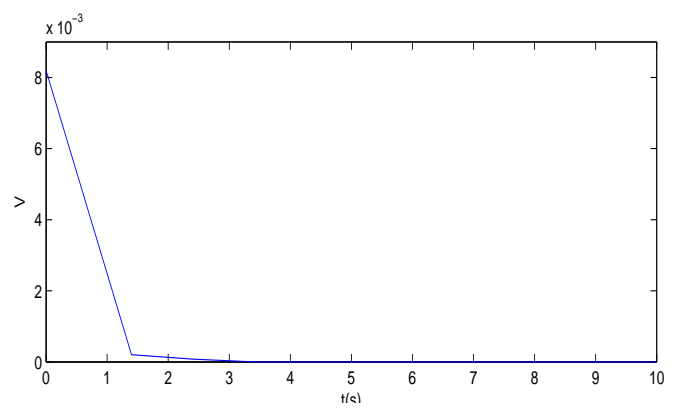

Fig. 9. Variation of the Lyapunov function

To facilitates the co-simulation of controller task execution in real-time kernels, network transmissions and continuous plant dynamics, we implement, a real time simulator, the control module Matlab/TRUE TIME [30].
The implementation in this environment consists in two phases. First of all, we perform the first three steps of Algorithm 1 offline. The last phase of this algorithm, which is the computation of the switching law, is made online: the determination of the sampling horizon is made progressively depending on the state.

Fig. 10 presents the triggers of the TRUETIME Send and TrueTime Receive blocs interconnected by the TRUE TIME Network. This figure shows the variation of sampling while the asymptotic convergence of the system state is illustrated in Fig. 11.

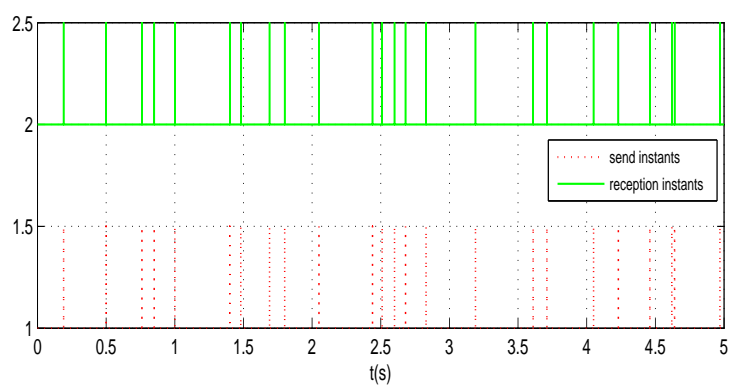

Fig. 10. Instants of information transition in the bloc TRUE TIME Network

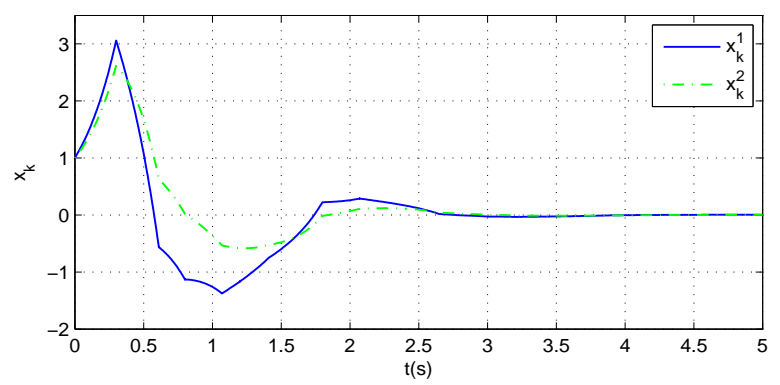

Fig. 11. Variation of the system state controlled in real time in the TRUETIME environment

Remark 4: We remark from Fig. 8 and Fig. 11 that, for the same initial conditions, the system state does not have the same behaviour. This can be explained by the occurrence of additional perturbations in the real-time system (process delay, actuation in real-time, ...) introduced by the TRUE TIME module for providing a more accurate simulation.

\section{Conclusion}

This paper has presented a technique for adapting the sampling law with regard to the present state value.

First, a cartography of the state space is defined on the basis of Lyapunov functions. Next, a dynamic scheduling algorithm is presented. This algorithm takes into account the position of the state in this cartography and achieves an online optimization of the sampling sequence over a finite horizon. A switched system model is being used. The overall stability study is proven by 
means of poly-quadratic Lyapunov functions fitted for switched discrete-time system in association with LMI techniques.

As a result, our strategy allows for reducing the number of sampling instants. For instance, the stability limit for the period in the classical, constant sampling rate-case, can be overpassed, as illustrated in the final example. A Matlab/TRUE TIME implementation shows that the results can be applied in concrete situations of real-time control.

For the future research, we envisage to quantify the performance of the system in the continuous time to guarantee a continuously decreasing quadratic Lyapunov function.

\section{REFERENCES}

[1] B. Wittenmark, J. Nilsson, and M. Torngren, "Timing problems in real-time control systems," Proceedings of the American Control Conference, pp. 2000-2004, 1995.

[2] X.-G. Li, A. Cela, S. Niculescu, and A. Reama, "Some problems in the stability of networked-control systems with periodic scheduling," International Journal of Control, vol. 83, pp. 996-1008, 2010.

[3] W. Zhang, M. Branicky, and S. Phillips, "Stability of networked control systems," IEEE Control Systems magazine, vol. 21, no. 1, pp. $84-99,2001$.

[4] J.-P. Richard and T. Divoux, Systeme commande en reseau. Hermes-Lavoisier, 2007.

[5] E. Fridman, A. Seuret, and J.-P. Richard, "Robust sampleddata stabilization of linear systems: an input delay approach," Automatica, vol. 40, no. 8, pp. 1441 - 1446, 2004.

[6] E. Fridman, "A refined input delay approach to sampled-data control," Automatica, vol. 46, no. 2, pp. 421 - 427, 2010.

[7] A. Seuret, "Stability analysis for sampled-data systems with a time-varying period," pp. $8130-8135$, december 2009 .

[8] L. Hetel, A. Kruszewski, W. Perruquetti, and J.-P. Richard, "Discrete and intersample analysis of systems with aperiodic sampling," IEEE Transactions on Automatic Control, vol. 56, no. 7, pp. $1696-1701$, july 2011.

[9] K. Gu and S. Niculescu, "Survey on recent results in the stability and control of time-delay systems," Journal of Dynamic Systems, Measurement, and Control, vol. 125, no. 2, pp. 158$165,2003$.

[10] K. Gu, V. Kharitoniv, and J. Chen, Stability of Time-Delay Systems. Boston: Birkhauser, 2003.

[11] J.-P. Richard, "Time-delay systems: an overview of some recent advances and open problems," Automatica, vol. 39, no. 10, pp. $1667-1694,2003$.

[12] W. Jiang and A. Seuret, "Improved stability analysis of networked control systems under asynchronous sampling and input delay," 2nd IFAC Workshop on Distributed Estimation and Control in Networked Systems, Annecy, France, p. 73, 2010.

[13] P. Naghshtabrizi, J. Hespanha, and A. Teel, "Stability of delay impulsive systems with application to networked control systems," Transactions of the Institute of Measurement and Control, vol. 32, no. 5, pp. 511-528, 2010.

[14] K. Liu and E. Fridman, "Stability analysis of networked control systems: A discontiuous lyapunov functional approach," 48th IEEE Conference on Decision and Control, Shanghai, China, pp. $1330-1335,2009$.

[15] P. Tabuada, "Event-triggered real time scheduling of stabilizing control tasks," IEEE Transactions on Automatic Control, vol. 52, no. 9, pp. 1680-1685, 2007.

[16] R. Cogill, S. Lall, and J. Hespanha, "A constant factor approximation algorithm for event-based sampling," in American Control Conference, july 2007, pp. 305-311.

[17] W. Heemels, J.-H. Sandee, and P. V. Bosch, "Analysis of event-driven controllers for linear systems," International Journal of Control, vol. 81, no. 4, pp. 571-590, 2008.
[18] J. Lunze and D. Lehmann, "A state-feedback approach to event-based control," Automatica, vol. 46, no. 1, pp. 211-215, 2010.

[19] M. Mazo and P. Tabuada, "Decentralized event-triggered control over wireless sensor/actuator networks," in IEEE Transactions on Automatic Control, vol. 56, 2011, pp. 2456-2461.

[20] X. Wang and M. Lemmon, "Self-triggered feedback control systems with finite-gain 12 stability," IEEE Transactions on Automatic Control, vol. 45, no. 3, pp. 452-467, 2009.

[21] D. Dimarogonas, E. Frazzoli, and K. Johansson, "Distributed self-triggered control for multi-agent systems," in IEEE Conference on Decision and Control, december 2010, pp. 6716 6721.

[22] X. Wang and M. Lemmon, "Self-triggering under stateindependent disturbances," IEEE Transactions on Automatic Control, vol. 55, no. 6, pp. 1494-1500, 2010.

[23] M. Mazo, A. Anta, and P. Tabuada, "An iss self-triggered implementation of linear controllers," Automatica, vol. 46, no. 8, pp. 1310-1314, 2010.

[24] C. Fiter, L. Hetel, W. Perruquetti, and J.-P. Richard, "A state dependent sampling for linear state feedback," in Automatica (Provisionally accepted), 2012.

[25] D. Liberzon, J. Hespanha, and A. Morse, "Stability of switched systems: a lie-algebraic condition," in Systems and Control Letters, vol. 37, 1999, pp. $117-122$.

[26] J.-W. Lee and G. Dullerud, "Uniform stabilization of discretetime switched and markovian jump linear systems," Automatica, vol. 42, no. 2, pp. $205-218,2006$.

[27] W. Zhang, A. Abate, J. Hu, and M. Vitus, "Exponential stabilization of discrete-time switched linear systems," in $A u$ tomatica, vol. 45,2009 , pp. $2526-2536$.

[28] P. Mason, M. Sigalotti, and J. Daafouz, "On stability analysis of linear discrete-time switched systems using quadratic lyapunov functions," in IEEE Conference on Decision and Control, december 2007, pp. $5629-5633$.

[29] H. Fujioka, "Stability analysis of systems with aperiodic sample-and-hold devices," Automatica, vol. 45, no. 3, pp. 771 $-775,2009$.

[30] P. Reutersward, J. Akesson, A. Cervin, and K.-E. Arzen, "Truetime network: A network simulation library for modelica," in Proceedings of the 7th International Modelica Conference. Modelica Association, 2009. 